\section{A Natural Pentaploid Strawberry Genotype from the Changbai Mountains in Northeast China}

\author{
Lei Jiajun ${ }^{1}$ \\ College of Horticulture, Shenyang Agricultural University, Shenyang \\ 110161,China
}

\section{Li Yuhua}

College of Landscape Architecture, Northeast Forestry University, Harbin 150040, China

\section{Du Guodong, Dai Hanping, and Deng Mingqin \\ College of Horticulture, Shenyang Agricultural University, Shenyang 110161,China}

Additional index words. strawberry, Fragaria, pentaploid, germplasm resources

\begin{abstract}
A natural strawberry genotype, 'Jilin 4', was collected from the Changbai Mountains located in Gongzhuling Region, Jilin Province, Northeast China. It was identified as a pentaploid $(2 n=5 x=35)$ by counting the chromosomes of the root-tip cells. Natural pentaploid strawberries have not been reported as originating in China or outside of California in the U.S. Jilin 4 was the most vigorous genotype among representatives of all 15 wild species of Fragaria and more than 60 cultivars of $F$. $\times$ ananassa in a common garden. The size of its flowers and anthers were similar to those of cultivars and it produced abundant runners. The pollen was viable, but the pistils were sterile. It appeared to be drought and cold tolerant, but showed symptoms of local virus infection. There are three possible origins of Jilin 4, which are discussed in this report.
\end{abstract}

Of about 20 recognized Fragaria species, 11 are distributed in China, including 8 diploid species-F. daltoniana J. Gay, F. vesca L., $F$. gracilis A. Los., F. mandschurica Staudt, $F$. nilgerrensis Schlect., F. nubicola Lindl., F. pentaphylla Lozinsk, and $F$. viridis Duch. - and 3 tetraploid species - F. corymbosa Lozinsk., $F$. moupinensis (French.) Card., and $F$. orientalis Lozinsk (Lei et al., 1999 and 2001; Staudt, 1999). We have collected wild strawberries throughout China since 1980, and to date, have obtained more than 120 individuals of 10 species. In a recent unpublished survey of 15 F. orientalis genotypes from the Provinces of Jilin, Inner Mongolia and Heilongjiang, we found that 10 were diploids $(2 n=14), 4$ were tetraploids $(2 \mathrm{n}=28)$ and 1 was a pentaploid $(2 n=35)$. The pentaploid, designated 'Jilin 4', was collected in 1989 from the forest border of Changbai Mountains near the Gongzhuling Region in the Jilin Province, northeastern China. Herein, we describe this pentaploid genotype and speculate on its origins.

\section{Materials and Methods}

'Jilin 4' was propagated by runners and maintained at the Strawberry Repository of Shenyang Agricultural University. Thirty-six plants were set in the field at $25 \times 25-\mathrm{cm}$ spacings in two blocks dispersed within a

Received for publication 21 May 2004. Accepted for publication 18 Sept. 2004. Thanks to Jim Hancock for editing and submitting the paper.

1'Author for correspondence; e-mail:jiajunlei@yahoo. com.cn. larger planting of native Fragaria clones from across China and the world. Within the field were planted several representatives of diploid F. gracilis, F. mandschurica, F. nilgerrensis, $F$. pentaphylla, $F$. vesca, and $F$. viridis, tetraploid $F$. corymbosa, F. moupinensis, and $F$. orientalis from China, diploid $F$. iinumae Makino, $F$. nipponica Lindl. and $F$. yezoensis Hara. from Japan, hexaploid F. moschata Duch. from Europe, and octoploid $F$. chiloensis (L.) Duch. and F. virginiana Duch. from the U.S. Also included in the field were 60 octoploid strawberry cultivars of $F$. ×ananassa from all across the world, and some artificially produced pentaploid hybrids of 'Honeoye' $\times F$. nilgerrensis, 'Honeoye' $\times F$. viridis and 'Honeoye' $\times F$. vesca. The height of 10 'Jilin 4'plants and their inflorescences was measured during full bloom.

Chromosomes were counted in at least 10 root-tip cells of 'Jilin 4' in 1994, 1995, and 1997 using the squash method. The root tips were pretreated for about $2 \mathrm{~h}$ with saturated 1,4-dichlorobenzene $\left(\mathrm{C}_{6} \mathrm{H}_{4} \mathrm{Cl}_{2}\right)$, subjected to $5 \mathrm{~mol} \cdot \mathrm{L}^{-1} \mathrm{HCl}$ for $3 \mathrm{~min}$ and stained with basic fuchsin $\left(\mathrm{H}_{2} \mathrm{NC}_{6} \mathrm{H}_{4}\left[\left(\mathrm{C}_{6} \mathrm{H}_{4} \mathrm{CNH}_{2} \mathrm{Cl}\right)\right.\right.$ $\left.\mathrm{C}_{6} \mathrm{H}_{3} \mathrm{CH}_{3} \mathrm{NH}_{2}\right]$ ) for 5 to $10 \mathrm{~min}$ (Lei, 1997).

Anthers were collected from several flowers of 'Jilin 4', the $F$. ×ananassa cultivars Honeoye and Toyonoca, and single representatives of $F$. viridis, $F$. vesca, $F$. nilgerrensis, and $F$. virginiana. The anthers were allowed to dehise on paper in an open room for $24 \mathrm{~h}$, and then the viability of 200 to 300 pollen grains of each plant was tested by staining them with benzidine-alpha-naphthol liquid (Shen, 1992). The fertility of 'Jilin 4' was further tested by pollinating emasculated flowers with fresh pollen from individuals of $F$. vesca, 'Honeoye' and 'Hokowase', and a pentaploid hybrid of 'Honeoye' $\times F$. vesca, both singly and in combination. 'Jilin 4' was also crossed as male parent with each of the same genotypes. Twenty to thirty flowers were crossed in each combination, and fruit set was measured as the number of flowers crossed divided by the number of flowers that produced fruit.

\section{Results}

Chromosome counts and fertility. 'Jilin 4' appeared to be a stable pentaploid $(2 \mathrm{n}=$ $5 \mathrm{x}=35$ ) in all 3 years (Fig. 1). The natural pentaploid had an average pollen viability of $64 \%$, compared to $78.2 \%, 88.5 \%$, and $91.7 \%$ for the individuals of $F$. viridis, $F$. virginiana and 'Honeoye'. No seeds were produced from 'Jilin 4' $\times F$. vesca, 'Jilin 4' $\times$ the artificial pentaploid ('Honeoye' $\times$ F. vesca), or 'Jilin 4' $\times$ 'Honeoye' and 'Hokowase', both singly and in combination. However, fruit set was $15.4 \%$ when 'Jilin 4' was crossed as a male parent with the pentaploid interspecific hybrid and $67.2 \%$ when crossed with 'Honeoye'. A small proportion ( $1 \%$ ) of 'Jilin 4' pollen were $1 / 3$ to $1 / 4$ size of normal strawberry pollen (Fig. 2), although most appeared to be in the size range of the diploids $F$. viridis, $F$. vesca, F. nilgerrensis, $F$. virginiana, and the $F$. ×ananassa cultivars 'Honeoye' and 'Toyonoka'.

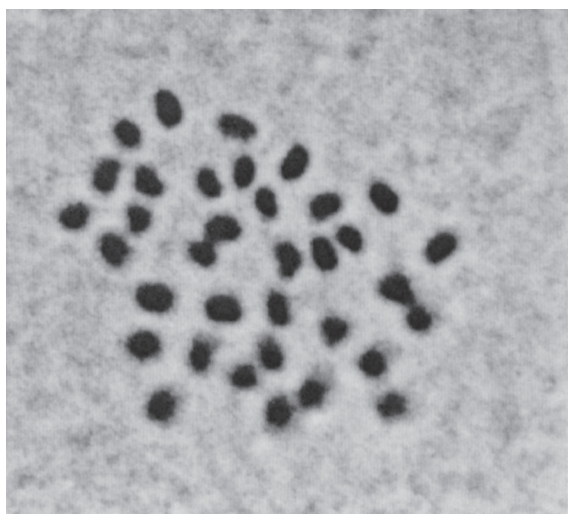

Fig. 1. The number of chromosomes in root-tip cells of the natural pentaploid strawberry genotype 'Jilin 4' $(2 \mathrm{n}=5 \mathrm{x}=35)$.
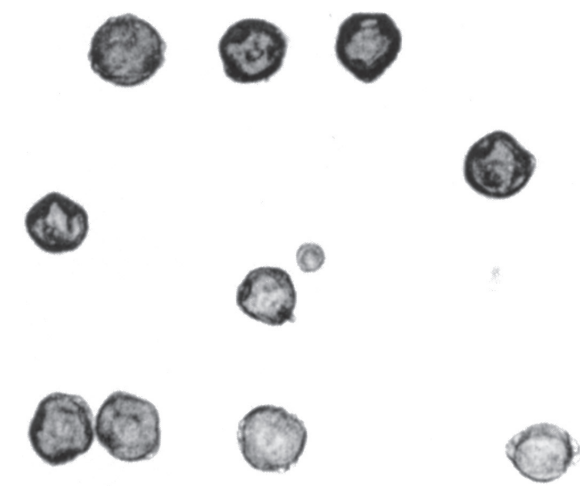

Fig. 2. Pollen grains of the natural pentaploid strawberry genotype 'Jilin 4 '. Note the unusually small grain in the center of the picture. 


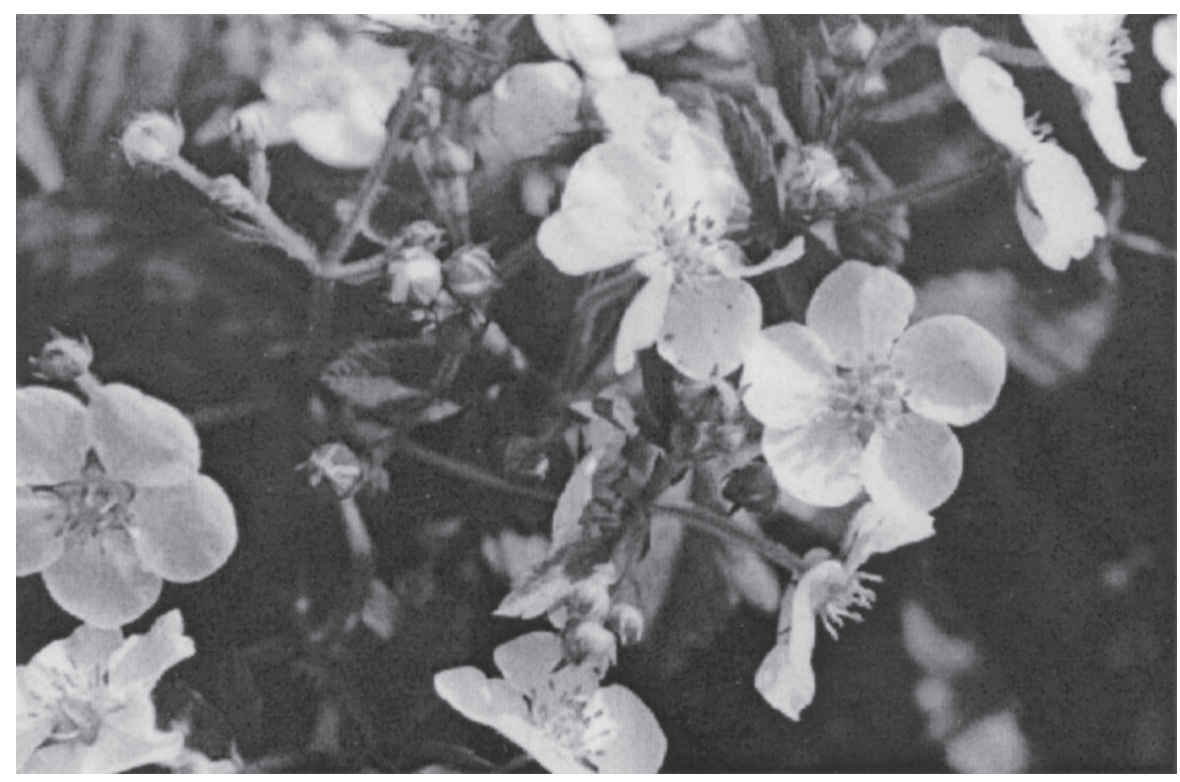

Fig. 3. The flowers of the natural pentaploid strawberry 'Jilin 4'.

Morphology. The wild strawberry genotype 'Jilin 4' appeared to us to much more closely resemble $F$. moschata, $F$. orientalis, and $F$. mandschurica, than the other strawberry species of China including $F$. gracilis , F. moupinensis, $F$. nilgerrensis, $F$. pentaphylla, and $F$. viridis. However, 'Jilin 4' was much taller than all the other species in the field ( 35 to $40 \mathrm{~cm}$ ), had larger leaves and flowers, and had a much more erect inflorescence (Fig. 3).

'Jilin 4' was unusually vigorous, compact and hairy. Its leaf was deeply serrated with sunken veins, the stipule sheath was deep red, and the center leaflet was rhombic. Runners were abundant with long internodes. Daughter plants formed at the even nodes, with the odd nodes producing either a second runner or a scaly leaf. The inflorescence was 40 to $45 \mathrm{~cm}$ in height, erect and thick, and much higher than leaf level. There were 4 to 10 inflorescences per plant with 7 to 25 flowers per inflorescence. Two leaflets were generally found at the fork of each inflorescence; however, a few plantlets with normal leaf and adventitious roots were produced at the fork, which is very uncommon in Fragaria. The flower size averaged $3.0 \mathrm{~cm}$, which was by far the largest of any of the wild species, and is nearly as large as the cultivars. It started to blossom on about 20 May in the open field in Shenyang Regions. The number of anthers in each flower of 'Jilin 4' was usually between 20 and 25 and the size of its anthers was about the same as a typical cultivar. The petals were almost round, folding, and usually 5 in number, although there were sometimes 6 or 7 . The calyx was triangular and the calycle was thin lanceolate. The receptacle was very small and pistils were extremely short. A few days after the flowers opened, the pistils became red and then brown, and then appeared to die. At petal fall, five filaments bent to the center of each flower and covered the pistils, which is also unusual in Fragaria. The pedicels bent downwards when the petals dropped. Ultimately, the flower and inflorescence withered.

'Jilin 4' was one of the few plants that thrived during extended periods of drought, and it survived the winter unusually well without mulch. However, it was highly susceptible to the local viruses strawberry crinkle and strawberry mosaic. Infected plants showed symptoms of dwarfing, crinkle, distortion and mosaic.

\section{Discussion}

Based on the geographical distribution of the natural pentaploid 'Jilin 4' and the other wild Fragaria species, three kinds of origins are possible.

1) Normal gametes of diploid $F$. mandschurica or $F$. vesca could have combined with unreduced gametes of tetraploid $F$. orientalis. In Fragaria, unreduced gametes are considered to be relatively common (Bringhurst and Gill, 1970), and we have previously identified a few unusually large pollen grains in $F$. orientalis that could have been unreduced (Lei, 1997).

2) Unreduced gametes of diploid $F$. mandschurica or $F$. vesca could have crossed with normal gametes of hexaploid $F$. moschata.

3 ) Normal unreduced gametes of tetraploid $F$. orientalis and hexaploid $F$. moschata could have combined.
With the information at hand, it is difficult to distinguish between these three possibilities as $F$. mandshurica, $F$. vesca, $F$ orientalis and $F$. moschata appear quite similar, and a previous RAPD analysis showed that the diploid Jilin 1 (F. mandschurica) and a tetraploid F. orientalis collected from Changbai Mountains were closely related to hexaploid $F$. moschata (Lei et al., 1999). Other types of molecular markers will have to be used to distinguish between the three possible origins of Jilin 4.

Until now, natural pentaploid hybrids of Fragaria have only been found in mixed populations of octoploid $F$. chiloensis and $\operatorname{diploid} F$. vesca in coastal California of the United States (Bringhurst et al., 1963; 1966), and have been named F. bringhurstii (Staudt, 1999). The natural pentaploid Jilin 4 distributed in Northeast China is derived from a unique combination of species and should prove to be a valuable new wild strawberry resource. It could play an important role in determining the phylogeny of Fragaria and may be an important source of genes for strawberry breeding. We have been using it for its cold tolerance, vigor and high pollen viability.

\section{Literature cited}

Bringhurst R.S. and D.A. Khan. 1963. Natural pentaploid Fragaria chiloensis-F. vesca hybrids in coastal California and significance in polyploid Fragaria evolution. Amer. J. Bot. 52:658-661.

Bringhurst R.S. and Y.D.A. Senanayake. 1966. The evolutionary significance of natural Fragaria chiloensis $\times F$. vesca hybrids resulting from unreduced gametes. Amer. J. Bot. 57:969-976.

Bringhurst, R.S. and T. Gill. 1970. Origin of Fragaria polyploids: Unreduced and double unreduced gametes. Amer. J. Bot. 53:1000-1006.

Lei, J.J. 1997. Studies on chromosome doubling and wide crosses in strawberry (in Chinese). PhD diss. Shenyang Agr. Univ.

Lei, J.J., G. Yang, H.P. Dai, L.P. Wu, and M.Q. Deng. 1997. The wild strawberry germplasm resources in China (in Chinese). J. Fruit Sci. 14:198-200.

Lei, J.J., T. Mochizuki, Y. Noguchi, and K. Sone. 1999. Phylogenetic analysis of Fragaria species using RAPD markers (in Japanese). J. Jpn. Soc. Hort. Sci. 68(Suppl. 2):133.

Lei, J.J., T. Mochizuki, and M.Q. Deng. 2001. Studies on the diploid strawberry species Fragaria mandschurica Staudt (in Chinese). J. Fruit Sci. 18:337-340.

Shen, D.X. 1992. Experiment technology of fruit breeding (in Chinese), p. 40-42. Agr. Publ. Co., Beijin.

Staudt, G. 1999. Systematics and geographical distribution of the American strawberry species: Taxonomic studies in the genus Fragaria (Rosaceae: Potentilleae). Univ. Calif. (Berkeley) Publ. Bot. vol. 81. 